\title{
UN MODELO DE ELECCIÓN DE MEDIDAS TRIBUTARIAS. EL CASO DE AMÉRICA LATINA
}

Victor Mauricio Castañeda Rodríguez*

En la fijación de tasas de impuestos y bases gravables inciden la es factible y puede constituir un equilibrio político-económico establecer tratos preferenciales y sistemas tributarios complejos, aunque no sean eficientes (Hettich y Winer, 1999, 1997, 1988). Así, la política tributaria es afectada por las preferencias de los políticos y sus intereses electorales, lo que los lleva a tener en cuenta la movilidad política de cada grupo o sector social, su nivel esperado de ingresos y la ponderación que da al gasto público en su bienestar.

E1 modelo que aquí se propone predice que la dependencia de los impuestos al consumo (al IVA, p. ej.) tiende a disminuir cuando las expectativas sobre el desempeño económico son favorables, en especial para las clases pobres y medias, porque para los políticos es electoralmente útil bajar sus tasas de impuestos, aunque la ampliación de sus bases gravables no compense la pérdida de recaudo. A su vez, es plausible aumentar la participación de impuestos como el IVA cuando mejora la percepción ciudadana acerca del gasto público, lo que significa que la relación entre el Estado y la sociedad no es solo de coerción sino también de cooperación (Torgler, 2005). La magnitud de los cambios de las tasas de impuestos que se proponen durante una reforma, en particular de las tasas al consumo, está supeditada al tamaño y la movilidad política de cada grupo.

\footnotetext{
* Doctor en Ciencias Económicas; profesor de la Universidad Jorge Tadeo Lozano, Bogotá, Colombia, [victorm.castanedar@utadeo.edu.co]. Fecha de recepción: 4 de junio de 2013, fecha de modificación: 12 de febrero de 2014, fecha de aceptación: 29 de abril de 2015. Sugerencia de citación: Castañeda R., V. M. "Un modelo de elección de medidas tributarias. E1 caso de América Latina", Revista de Economía Institucional 17, 32, 2015, pp. 157-181. DOI: 10.18601/01245996.v17n32.05
} 
Estas consideraciones ayudan a explicar la gran heterogeneidad de la estructura tributaria entre países. En América Latina, los países más desarrollados suelen presentar una mayor participación de los impuestos directos, lo que a veces se explica señalando que el PIB per cápita es una proxy del desarrollo institucional y que, por ello, capta la fortaleza del Estado para recaudar esos impuestos (Besley y Persson, 2009). Este trabajo explica ese fenómeno de otro modo, como una decisión racional de los políticos de bajar (elevar) los impuestos al consumo de los grupos sociales con menor preferencia por el futuro -los grupos pobres y medios- cuando la economía muestra un comportamiento favorable (desfavorable).

Si bien el modelo incorpora elementos políticos como la influencia de los votantes, según su pertenencia a algún grupo, la comprobación o constatación empírica solo se refiere al ámbito macroeconómico de la tributación, y no se analiza la variación de las tasas de impuestos sino el comportamiento de la estructura y la presión tributaria (PT); por ello recurre a algunos supuestos y proposiciones para determinar el comportamiento de la participación del IVA, entre otras cosas. En el ejercicio econométrico se usa un panel de datos no balanceado que cubre 18 países de América Latina, en el periodo 1980-2010, e incluye algunas variables de control para evitar problemas de especificación, conforme a la revisión de los trabajos empíricos pertinentes que hacen Castañeda (2012) y Dioda (2012).

En la primera parte del artículo se estudia el problema de elección de un vector tributario, integrado por las tasas aplicables al consumo $\mathrm{y}$ al ingreso de cada contribuyente (según el grupo de votantes al que pertenezca), que enfrenta un candidato a un cargo público con poder de iniciativa tributaria (como el presidente). En la segunda se constata el modelo con datos de América Latina y al final se presentan las conclusiones.

\section{LA DECISIÓN DE POLÍTICA TRIBUTARIA}

El sistema tributario de un país o región, pese a su complejidad y desorden, es un resultado de decisiones racionales de contribuyentes y políticos y puede entonces ser un equilibrio político-económico ${ }^{1}$. Si bien algunos factores económicos limitan las opciones de política tributaria, los políticos proponen reformas particulares no necesariamente basadas en objetivos como reducir los costos de administra-

1 Ver Castanheira et al. (2012), Winer y Hettich (2003), Hettich y Winer (1999, 1997, 1988) y Lindbeck y Weibull (1987). 
ción, lograr una mayor eficiencia o mejorar la progresividad de los impuestos.

De hecho, las iniciativas de política tributaria son afectada por las posibles respuestas de los diferentes grupos de votantes a esas iniciativas (Castanheira et al., 2012), de modo que los sistemas tributarios pueden introducir amplias distorsiones, aun en equilibrio, dependiendo de la movilidad electoral de tales grupos (Winer, 2002; Galli y Profeta, 2007). En otras palabras, las motivaciones sociales -redistributivas, p. ej.- no necesariamente son plenamente internalizadas por la tributación.

Así, un político con poder de iniciativa tributaria no incurre en el costo electoral de mejorar el bienestar de todos los votantes si puede identificar a los más sensibles a sus propuestas. No es extraño que los políticos y sus partidos, de izquierda o de derecha, tiendan a proponer proyectos similares para atraer votantes indecisos, pues cuentan con una base electoral relativamente cautiva.

Aquí se propone resolver el problema estándar de la elección de política tributaria usando un modelo de votación probabilística ${ }^{2}$, pues el enfoque del votante mediano tiene problemas, como la inexistencia de equilibrios de Nash, cuando la votación es por mayorías y el espacio de decisión es multidimensional (Persson y Tabellini, 2000).

Sin embargo, primero se debe considerar que en la práctica una reforma tributaria se refiere a cualquier modificación de los parámetros del sistema impositivo, como tarifas, bases y tratos diferenciales (Hettich y Winer, 1988). Es decir, una reforma no solo se manifiesta en la aprobación de una ley, pues los cambios de reglamentación o de medidas adoptadas por la administración de impuestos también inciden en la tasa efectiva de tributación. Por ello, en el modelo que se presenta enseguida, las tarifas óptimas no necesariamente deben ser aprobadas por el congreso, pues según su alcance se pueden aplicar mediante medidas que competen al gobierno.

Para nuestro propósito los electores se dividen en $\mathrm{H}$ grupos, de tamaño normalizado igual a $\alpha_{h}=n_{h} / n(h: 1 \ldots, \mathrm{H})$, donde $n_{b}$ es el número de contribuyentes del grupo $h$ y $n=\sum_{h=1}^{H} n_{b}$. Además, conforme a la hipótesis del ingreso permanente (Friedman, 1957), los individuos enfrentan una restricción presupuestal intertemporal y el valor presente de su consumo en el ciclo de vida es igual al de sus ingresos más su capital inicial ${ }^{3}$. Así, el consumo de cada periodo es menos sensible a cambios

${ }^{2}$ Como proponen Profeta (2007), Coughlin (1992) y Lindbeck y Weibull (1987).

${ }^{3}$ También se puede suponer que el valor presente del consumo en un periodo $T$ es una fracción del presupuesto disponible sin que cambie el resultado de esta sección. 
tributarios temporales, lo que es razonable dada la existencia de un mercado de crédito y de unos patrones de consumo, aunque los contribuyentes perciban mayor desutilidad de los impuestos indirectos.

La función de utilidad de un elector del grupo $h$ en el periodo $t\left(U_{b}\right)$, con un consumo $C_{b t}$ un gasto público $G_{t}$ y un índice de ponderación social de la utilidad del gasto $\beta \in(0,1]$, adopta la forma:

$U_{b t}=\ln C_{h t}+\beta G_{t}$

Los ciudadanos son, entonces, aversos al riesgo en materia de consumo, lo que es coherente con la práctica de establecer un patrón de consumo y no determinarlo únicamente por el ingreso corriente. La restricción presupuestal de un votante del grupo $h$, con la información disponible en el periodo inicial $\left(\mathrm{E}_{0}\right)$, la tasa de interés común a toda la economía, las tasas de impuestos de renta y de consumo $t_{r b}$ y $t_{c b}$ en $t=0$, un ingreso $y_{b t}$ en $t \mathrm{y}$ un capital inicial $K_{b 0}$ es:

$\sum_{t=0}^{T}\left(\frac{1}{1+r}\right)^{t} \mathrm{E}_{0} C_{b t}=\frac{\left(1-t_{t b}\right) \sum_{t=0}^{T}\left(\frac{1}{1+r}\right)^{t} \mathrm{E}_{0} y_{b t}+K_{b 0}}{1+t}$

Como los impuestos afectan las decisiones económicas -de inversión y del esfuerzo para obtener ingresos-, $y_{b t}$ depende, entre otros factores, de la tasa del impuesto de renta. Por simplicidad se supone que la relación entre $y_{b t}$ y $t_{r b}$ es lineal para cada grupo, $y_{b t}^{\prime}=\frac{\partial y_{b t}}{\partial t_{t h}}=\varepsilon_{b}<0$; además $t_{c b}$ limita el consumo según (2). Así, un alza de impuestos implica el aumento del recaudo sobre la base efectiva, pero también contrae esta última por la distorsión que genera; la diferencia entre la magnitud de estos efectos determina si la economía está en la sección creciente o decreciente de la curva de Laffer.

La decisión de consumo actual (en $t=0$ ) para un agente del grupo $b$ está dada por (3), donde $\delta_{b}$ es la tasa de descuento intertemporal y Eyh $=\sum_{t=0}^{T}\left(\frac{1}{1+\mathrm{r}}\right)^{t} \mathrm{E}_{0} y_{b t}$. Según este resultado, una reducción marginal del consumo en el presente $(\Delta C)$ para aumentarlo en el futuro no tiene efecto en la utilidad esperada del individuo, si es racional ${ }^{4}$.

$C_{b 0}=\frac{\left(1-t_{r b}\right) \mathrm{E}_{\mathrm{h}}+K_{b 0}}{\left(1+t_{c b}\right) \sum_{t=0}^{T}\left(\frac{1}{1+\delta_{b}}\right)^{t}}$

La función de utilidad indirecta de un votante del grupo $h$ en $t=0$, que se obtiene al remplazar (3) en (1), es:

$V_{b 0}=\ln \frac{\left(1-t_{r b}\right) \mathrm{Ey}_{\mathrm{h}}+K_{b 0}}{\left(1+t_{c b}\right) \sum_{\mathrm{t}=0}^{T}\left(\frac{1}{1+\delta_{b}}\right)^{\mathrm{t}}}+\beta G_{o}$

${ }^{4} \mathrm{Si}$ en cada periodo el ahorro en $t=0$ tiene un rendimiento igual a la tasa de interés $r, \mathrm{E}_{0} C_{b t}=\left(\frac{1+r}{1+\delta_{b}}\right)^{t} C_{b 0}$. Basta reemplazar esto en (2) para verificar (3).

Revista de Economía Institucional, vol. i7, n.o 32 , Primer semestre/2oi5, pp. i57-i 8 i 
Ahora debemos resolver el problema de un político que tiene intereses electorales, en particular que busca un cargo público con poder de iniciativa tributaria ${ }^{5}$. Para simplificar, supongamos que solo hay dos candidatos, $A$ y $B$, que no cooperan y consideran exógena la estrategia del otro. La probabilidad de ser elegidos no solo depende de sus propuestas económicas, sino también de las preferencias políticas de los electores de cada grupo y de la actitud ideológica de la sociedad. Si $\phi_{i b}$ y $\rho$ representan la preferencia de un votante $i$ del grupo $b$ por el político $A$ y la actitud ideológica general, respectivamente, $i$ votará por $A$ en $t=0 \mathrm{si}^{6}$ :

$V_{b 0}\left(t^{A}\right)-V_{b 0}\left(t^{B}\right)+\phi_{i b}+\rho>0$

donde $t=\left(t_{c 1}, \ldots, t_{c H}, t_{r 1}, \ldots, t_{r H}\right)$ es un vector de política tributaria y el superíndice indica el político que lo propone.

Esto implica que los candidatos tienen la voluntad de cumplir sus promesas, lo que es razonable si las instituciones políticas protegen el derecho de los votantes a destituir a los funcionarios elegidos que incumplen sus promesas de campaña sin justificación y hay participación ciudadana, por ejemplo mediante veedurías. Aunque se consideran las tasas impositivas al ingreso total y al consumo de cada contribuyente, estas se pueden representar por el promedio ponderado de las tasas diferenciales establecidas para cada una de las clasificaciones de la respectiva base gravable (p. ej., ingresos laborales y rentas de capital). Ningún político conoce el valor de las preferencias políticas, solo conoce su distribución de probabilidades. Siguiendo a Profeta (2007) y a Persson y Tabellini (2002), se supone que $\phi_{i h}$ y $\rho$ se distribuyen de modo uniforme en los intervalos $\left[-\frac{1}{2 \phi_{2}}, \frac{1}{2 \phi_{b}}\right]$ y $\left[-\frac{1}{2 \psi}, \frac{1}{2 \psi}\right]$ y respectivamente, con valores esperados iguales a 0 y densidades y $\Psi$. Los grupos difieren en la densidad de las preferencias idiosincráticas de sus integrantes. En suma, la probabilidad de que un elector del grupo $b$ vote por $A$ está dada por:

$\pi_{b}^{\mathrm{A}}=\int_{-\mathrm{V}_{b 0}}^{\frac{1}{2 \phi_{b}}}\left(t^{\mathrm{A}}\right)+V_{b 0}\left(t^{B}\right)-\rho^{\phi_{b}} d \phi_{b}=\frac{1}{2}+\phi_{b}\left[\mathrm{~V}_{b 0}\left(t^{\mathrm{A}}\right)-V_{b 0}\left(t^{B}\right)+\rho\right]$

Y la probabilidad de que se vote por A, agregando por grupos, es:

$\pi^{\mathrm{A}}=\sum_{h=1}^{H} \alpha_{h} \pi_{b}^{\mathrm{A}}$

${ }^{5}$ Esto no excluye la búsqueda de reelección.

${ }^{6}$. El problema de $B$ es idéntico. En adelante, se supone que los candidatos eligen su vector de políticas en el presente.

${ }^{7}$ Los electores del grupo cuyo es tal que el lado izquierdo de (5) es igual a 0 se llaman votantes pivotales, y los candidatos enfocan en ellos sus propuestas por ser los más sensibles. 
El problema de $A$ es maximizar la probabilidad de obtener más de la mitad de los votos; su función objetivo es la probabilidad de que (7) sea mayor que $1 / 2$. Si $\phi=\sum_{b=1}^{H} \alpha_{b} \phi_{b}$ es la densidad promedio de las preferencias políticas, y dado que $\rho$ se distribuye de modo uniforme con una densidad $\psi$, la función a maximizar es:

$P\left[\sum_{b=1}^{H} \alpha_{b} \phi_{b} \rho>-\sum_{b=1}^{H} \alpha_{b} \phi_{b}\left[\mathrm{~V}_{b 0}\left(t^{\mathrm{A}}\right)-\mathrm{V}_{b 0}\left(t^{\mathrm{B}}\right)\right]\right]=\frac{1}{2}+\frac{\psi}{\phi_{h=1}^{H}} \alpha_{b} \phi_{b}\left[\mathrm{~V}_{b 0}\left(t^{\mathrm{A}}\right)-\mathrm{V}_{b 0}\left(t^{\mathrm{B}}\right)\right]$

Remplazando (4) en (8), $A$ debe entonces:

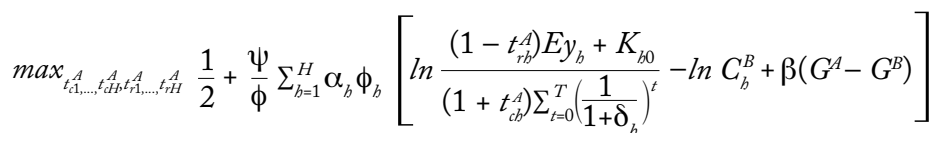

$$
\begin{aligned}
& \text { s.t. } G^{A}=\sum_{b=1}^{H} \alpha_{b}\left(t_{c b}^{A} \frac{\left(1-t_{r b}^{A}\right) E_{b}+K_{b 0}}{\left(1+t_{c b}^{A}\right) \Sigma_{t=0}^{T}\left(\frac{1}{1+\delta_{b}}\right)^{t}}+t_{r b}^{A} y_{b 0}\right)
\end{aligned}
$$

La ecuación (10), la restricción presupuestal del gobierno, define el gasto público como la suma, entre grupos de votantes, del recaudo de impuestos al consumo y de renta.

Una aproximación más realista exige corregir el consumo y el ingreso considerando la posibilidad de evasión; esto se puede hacer reduciendo $\beta$, lo que en la función de utilidad del individuo tiene un efecto similar al de una caída de la provisión pública de bienes y servicios. Así se hace aquí para no complicar más el modelo introduciendo variables que capten la capacidad institucional para recaudar impuestos; un elemento que ha sido estudiado entre otros autores por Besley y Persson (2010, 2009).

Una vez se reemplaza el gasto público en la función objetivo de $A$, las condiciones de primer orden para las tasas $t_{c b}^{A} y t_{r b}^{A}$ son:

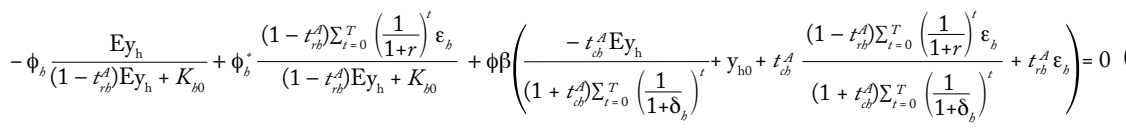

$$
\begin{aligned}
& \phi_{b}-\phi \beta \frac{\left(1-t_{t b}^{A}\right) \mathrm{Ey}_{\mathrm{h}}+K_{b 0}}{\left(1+t_{b b}^{A} \sum_{t=0}^{T}\left(\frac{1}{1+\delta_{b}}\right)^{t}\right.}=0
\end{aligned}
$$

Despejando $\phi_{b}$ de (12) y remplazándolo en (11), se obtiene:

$$
-\frac{\mathrm{Ey}_{\mathrm{h}}}{\sum_{t=0}^{T}\left(\frac{1}{1+\delta_{b}}\right)^{t}}+\frac{\left(1-t_{r b}^{A}\right) \sum_{t=0}^{T}\left(\frac{1}{1+r}\right)^{t} \varepsilon_{b}}{\sum_{t=0}^{T}\left(\frac{1}{1+\delta_{h}}\right)^{t}}+y_{b 0}+t_{r b}^{A} \varepsilon_{b}=0
$$


$\mathrm{Si} \prod_{b}=\sum_{t=0}^{T}\left(\frac{1}{1+r}\right)^{t}-\sum_{t=0}^{T}\left(\frac{1}{1+\delta_{b}}\right)^{t}$ es un índice de la diferencia relativa entre la tasa de interés y la tasa de descuento intertemporal del grupo $h$, despejando $t_{r b}^{A}$ de (13) para usar el resultado en (12) se obtiene:

$t_{r h}^{A}=\frac{\sum_{t=0}^{T}\left(\frac{1}{1+r}\right)^{t}}{\prod_{b}}-\frac{E_{\mathrm{h}}}{\prod_{b} \varepsilon_{b}}$

$t_{c b}^{A}=\frac{\beta}{\frac{\phi_{b}}{\phi} \sum_{t=0}^{T}\left(\frac{1}{1+\delta_{b}}\right)^{t}}\left[K_{b 0}+\frac{\left(\mathrm{E}_{\mathrm{h}}-\sum_{t=0}^{T}\left(\frac{1}{1+\delta_{b}}\right)^{t} \varepsilon_{b}\right) \mathrm{Ey}_{\mathrm{h}}}{\prod_{b} \varepsilon_{b}}\right]-1$

E1 resultado de la ecuación (14) sugiere tasas de impuesto de renta que tienden a ubicarse en los extremos del rango admisible: $[0,1]$. Si cada grupo de votantes tiene su tasa de descuento intertemporal particular, porque la aversión al riesgo afecta sus preferencias, y tiende a ser mayor en sectores pobres dadas sus necesidades básicas insatisfechas ${ }^{8}$, los grupos $h$ con $\delta_{b}>r\left(\prod_{b}>0\right)$ serán gravados con altas tasas de impuesto de renta (ISR). Para los grupos en mejor posición económica, con $\delta_{b}<r\left(\prod_{b}<0\right)$, la tasa sería mínima. Es decir, se tendería a adoptar un sistema tributario regresivo, un resultado teórico que se vuelve más factible cuando se considera que los electores de mayor poder económico suelen tener poder político y capacidad para incidir en las decisiones de los gobernantes y de los funcionarios elegidos a través del cabildeo. De allí el trato preferencial a las rentas de capital.

Esto no implica que la contribución de los pobres al ISR sea mayor en términos absolutos que la de los ricos, pues si bien estos últimos se benefician de tasas menores, tributan sobre una base mayor. Sean dos individuos $i \mathrm{y} j$; $i$ es un asalariado que gana 1.000 unidades monetarias (UM), $j$ recibe un ingreso laboral de $500 \mathrm{UM}$ y rentas de capital por 3.500 UM. Si los ingresos laborales se gravan al 30\% y las rentas de capital al 10\%, $i$ pagará 300 UM y $j 500$ UM en impuestos, de modo que $t_{r i}=30 \%$ y $t_{r j}=12,5 \%$.

E1 modelo condiciona el gasto público al ingreso fiscal, es decir, $G$ es una variable endógena; pero si se considera exógena, por su inflexibilidad en el corto plazo, la brecha entre las tasas máxima y mínima del ISR dependerá del compromiso presupuestal e incluso de $\beta$, pues si la capacidad fiscal del Estado es insuficiente o la sociedad no valora

\footnotetext{
${ }^{8}$ Esta idea es parte de la crítica de González y Pecha (2000) a los modelos neoclásicos de crecimiento, que supone una tasa de descuento intertemporal invariable.
} 
la acción estatal, es más difícil aumentar los impuestos al consumo y se sigue dependiendo del ISR.

Los resultados sugieren además que la posibilidad de aumentar la participación del ISR depende de que las diferencias socioeconómicas no sean muy grandes (menor desigualdad), lo que ayuda a explicar por qué los países desarrollados (OCDE, sin incluir México ni Chile) suelen depender más de este tipo de impuestos que los de América Latina (Tanzi y Zee, 2000). Puesto que $t_{r b}^{A}$ no depende de factores políticos, a diferencia de $t_{c b}^{A}$, el recaudo de impuestos directos está supeditado a las condiciones materiales de los contribuyentes, resultado que se basa en la hipótesis de ingreso permanente adoptada en la elaboración del modelo.

En el resultado de la ecuación (15) también influye la diferencia entre $r$ y $\delta_{b}$, de modo que hay una motivación para bajar la tasa del impuesto al consumo ceteris paribus a medida que la tasa de descuento intertemporal es menor. Además, la influencia política relativa del grupo $h,\left(\frac{\phi_{b}}{\phi}\right)$, se relaciona inversamente con $t_{c b}^{A}$, lo que significa que para los políticos es electoralmente útil reducir los impuestos al consumo de los grupos con mayor proporción de votantes indecisos. Por su parte, el acervo de capital compensa en parte la incidencia de la influencia política, pues el impuesto al capital es una fuente potencial de aumento del recaudo tributario. La ecuación (15) también sugiere que si el gobierno o los políticos perciben un deterioro de la situación económica, buscarán elevar las tasas efectivas de impuestos al consumo de los más impacientes (con $\prod_{b}>0$ ), lo que parece ser recurrente en las reformas tributarias realizadas en América Latina en tiempos de crisis, a juzgar por los cambios de la estructura tributaria desde los años ochenta, después de la crisis de la deuda externa (Castañeda, 2012). Los resultados anteriores se pueden sintetizar en dos proposiciones.

Proposición 1: la determinación políticamente óptima de las tasas relativas del impuesto de renta depende del grado de impaciencia de los votantes. Los grupos más impacientes tienden a ser gravados con tasas más altas. Pero si el gasto público se fija exógenamente, se limita la adopción de este tipo de políticas ${ }^{9}$.

9 Si se toma el lado derecho de la ecuación (14), su signo depende de $\Pi_{b}$. $\mathrm{Si}$ este parámetro es mayor (menor) que $0, \delta_{b}>\mathrm{r}\left(\delta_{b}<r\right)$, el primer término, que es $\frac{\sum_{t=0}^{T}\left(\frac{1}{1+r}\right)^{t}}{\Pi_{b}} \approx \frac{\delta_{b}(1+r)}{\delta_{b}-r}$ para un periodo suficientemente largo, será mayor que 1 (menor que 0), y como el segundo término es negativo (positivo), se tiene que $t_{r b}^{A}>1\left(t_{r b}^{A}<0\right)$. Así, la solución está condicionada por las restricciones que operan sobre las tasas de impuestos. 
Cabe señalar que si el gasto es endógeno, es de esperar un menor recaudo por ISR, dado el desincentivo económico del impuesto de renta. Los grupos más ricos prácticamente no tributarían, y el cobro a los más pobres frenaría sus iniciativas productivas; así, el gasto público se financiaría con impuestos al consumo pagados por los ricos $\left(\operatorname{con} \delta_{b}\right.$ $<r$ ); como sucedía en Cuba hasta la caída del bloque socialista, donde la relación entre el recaudo por impuestos generales al consumo y por ISR era de 388 en 1990 (Castañeda, 2012). Si el gasto es exógeno, no es posible que $t_{r b}^{A}=0$ ni $t_{r b}^{A}=1(\forall h)$, pero el impuesto seguirá siendo regresivo.

Proposición 2: $t_{c b}^{A}$ tiene una relación directa con el acervo de capital $\left(K_{b 0}\right)$ y el grado de impaciencia del grupo respectivo $\left(\Pi_{b}\right)$, y una relación inversa con la movilidad política $\left(\frac{\phi_{b}}{\phi}\right)$. Para los contribuyentes de los grupos con $\prod_{b}>0\left(\Pi_{b}<0\right)$, la tasa del impuesto al consumo es contra cíclica (cíclica), y el signo del efecto de la valoración del gasto público sobre ella depende del rango en que se ubique $\prod_{b}^{10}$.

Un hallazgo curioso es la relación inversa entre la tasa del impuesto al consumo de un grupo y el desempeño económico que esperan sus miembros cuando pues implica una política contracíclica. La intuición subyacente es que en una recesión los ingresos pueden caer más que el consumo, pues este depende del ingreso personal esperado en un periodo $T$ y no solo del nivel presente; lo que, sumado a la tendencia a reducir los impuestos al consumo de los grupos más pacientes (p. ej., a los bienes de lujo), impulsa al gobierno a tomar medidas para evitar que la PT caiga abruptamente, fijando tasas mayores al consumo de las clases pobre y media.

Pero queda la duda de si esto es electoralmente útil. Por ello, en la función de utilidad de los contribuyentes se incluye el efecto del gasto financiado, pues por su linealidad un aumento se asocia con una utilidad marginal mayor que la de un aumento del consumo privado de la misma magnitud, si $\beta$ toma valores suficientemente altos. De hecho, es difícil que el consumo crezca repentinamente porque sigue un patrón, que no solo depende del ingreso corriente; pero su reducción se puede compensar con gasto público, aún más si se logra mejorar su valoración social.

Algo más que merece atención es que este modelo describe el tipo de políticas que propondría un candidato con iniciativa tributaria;

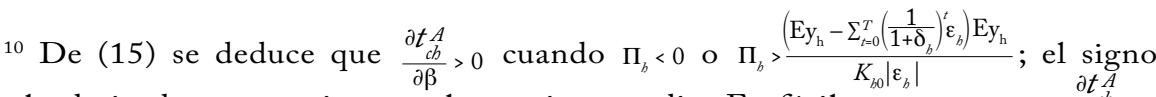
de la derivada es negativo en el caso intermedio. Es fácil constatar que $\frac{\partial t_{b} A}{\partial K_{b 0}}>0$ $\frac{\partial t_{c b}^{A}}{\partial \Pi_{b}}>0, \frac{\partial t_{c b}^{A}}{\partial \frac{\phi_{s}}{\phi}}<0$, y $\frac{\partial t_{c b}^{A}}{\partial \mathrm{E}_{\mathrm{h}}}<(>) 0$, esto último cuando $\Pi_{b}>0\left(\Pi_{b}<0\right)$.

Revista de Economía Institucional, vol. i7, N.o 32, Primer semestre/2oi5, Pp. I57-i 8 i 
pero cuando es elegido (como presidente, p. ej.) quizá no encuentre el apoyo necesario en el legislativo, pues el presidente se beneficia con la creación de nuevos programas de gasto y los congresistas pueden ser juzgados por el aumento de impuestos, sin recibir el crédito por lo que se financia. Por tanto, es necesario que el presidente tenga suficiente poder político y liderazgo en su partido para que se aprueben las políticas mencionadas, si para ello no es suficiente el cambio de la reglamentación o la administración tributaria.

Hasta ahora se han examinado los factores que afectan la selección de las tasas de impuestos, pero aún no es clara su relación con la dinámica tributaria a nivel agregado. No obstante, se pueden anticipar algunos efectos de los cambios de parámetros como el desempeño esperado de la economía o la valoración del gasto. Por ejemplo, es razonable que el aumento de las tasas de impuestos al consumo durante una recesión induzca un mayor recaudo, lo que junto a la menor base del ISR implica un aumento de la participación de esos impuestos en la PT. Para que esto sea cierto la economía debe estar en la sección creciente de la curva de Laffer.

Aunque no se presenta una prueba formal de esta afirmación, pues habría que hallar las raíces de un polinomio de quinto grado para la variable, se puede analizar la relación entre estructura tributaria y ponderación social del gasto público usando el modelo teórico, lo que es más fácil y permite exponer brevemente el método empleado para asociar y la dinámica impositiva. Para ello se formula la proposición 3.

Proposición 3: una mayor valoración social del gasto público permite aumentar el recaudo con impuestos al consumo de los grupos de votantes con menor grado de impaciencia $\left(\Pi_{b}<0\right)$. Para los más impacientes $\left(\Pi_{b}>0\right)$, este resultado depende de que el valor esperado de su ingreso permanente no sea "muy alto"11.

11 Primero se determina el recaudo por impuestos al consumo de un grupo particular, que corresponde a la expresión $C_{b} t c$. Si se remplaza cada término según las ecuaciones (3) y (15), se obtiene:

$C_{b} t_{c b}=\frac{\left(1-t_{t b}\right) \mathrm{Ey}_{\mathrm{h}}+K_{b 0}}{\sum_{t=0}^{T}\left(\frac{1}{1+\delta_{\mathrm{h}}}\right)^{t}}\left(1-\frac{\Pi_{b} \varepsilon_{b} \frac{\phi_{b}}{\phi} \sum_{t=0}^{T}\left(\frac{1}{1+\delta_{\mathrm{h}}}\right)^{t}}{\beta\left[K_{b 0} \Pi_{b} \varepsilon_{b}+\left(\mathrm{E}_{\mathrm{h}}-\sum_{t=0}^{T}\left(\frac{1}{1+\delta_{\mathrm{h}}}\right)^{t} \varepsilon_{b}\right) \mathrm{Ey}_{\mathrm{h}}\right]}\right)$

Ahora se considera cómo se comporta $C_{b} t_{c b}$ ante un cambio de $\beta$; por ello no es necesario expresar $t_{r b}$ según (14), pues no depende de $\beta$. Así:

$\frac{\partial C_{b} t_{c b}}{\partial \beta}=\frac{\left(1-t_{r b}\right) \mathrm{Ey}_{\mathrm{h}}+K_{b 0}}{\sum_{t=0}^{T}\left(\frac{1}{1+\delta_{\mathrm{h}}}\right)^{t}} \frac{\Pi_{b} \varepsilon_{b} \frac{\phi_{b}}{\phi} \sum_{t=0}^{T}\left(\frac{1}{1+\delta_{\mathrm{h}}}\right)^{t}}{\beta^{2}\left[K_{b 0} \Pi_{b} \varepsilon_{b}+\left(\mathrm{Ey}_{\mathrm{h}}-\sum_{t=0}^{T}\left(\frac{1}{1+\delta_{\mathrm{h}}}\right)^{t} \varepsilon_{b}\right) \mathrm{Ey}_{\mathrm{h}}\right]}$

E1 primer factor del lado derecho de esta expresión es positivo; para que una mayor valoración social del gasto lleve a un aumento del recaudo por el consumo del grupo $h$ se requiere que el segundo término también sea positivo. Si $\Pi_{b}<0$, el 
La proposición anterior plantea un problema, el valor esperado de los flujos de ingresos de un contribuyente con baja disposición a esperar $\left(\Pi_{b}>0\right)$ podría estar fuera del rango mencionado en la nota 11 , aunque es poco probable si el efecto distorsión $\left(\varepsilon_{b}\right)$ es grande. Asimismo, si el desempeño económico es regular para los grupos sociales más vulnerables, o con menor preferencia por el futuro, tiende a situarse entre esos límites, de modo que en periodos de crisis la mejor valoración del gasto público contribuye asimismo a aumentar los impuestos al consumo.

\section{CONSTATACIÓN EMPÍRICA CON DATOS DE PANEL}

Una opción para comprobar los resultados del modelo teórico es considerar una reforma tributaria particular y contextualizarla, según las circunstancias del momento, lo que requiere datos de encuestas, en especial durante elecciones, cuando se pueden relacionar las preferencias de voto con las propuestas de los candidatos, como hizo Profeta (2007) para las elecciones presidenciales de Italia en 2004. Otra opción, indirecta, es analizar la evolución de la estructura tributaria de uno o varios países, y establecer si hay alguna relación con variables agregadas que capten elementos del modelo, aunque esto implica desatender otros por falta de información.

En esta sección se opta por la segunda opción, que si bien no internaliza las preferencias políticas de los votantes, examina variables macroeconómicas que afectan sus decisiones como contribuyentes y las políticas que promueven agentes con iniciativa tributaria, de modo que confluyen las decisiones de fijación óptima de tasas de impuestos y las condiciones en que se aplican. Se considera el caso latinoamericano usando un panel de datos no balanceado del periodo 1980-2010 de 18 países $^{12}$.

numerador y el denominador son positivos, pues $\varepsilon_{b}<0$, lo que prueba la primera parte de la proposición.

Si $\Pi_{b}>0$, es necesario que $K_{b 0} \Pi_{h} \varepsilon_{\mathrm{h}}+\left(\right.$ Eyh $\left.-\sum_{t=0}^{T}\left(\frac{1}{1+\delta_{\mathrm{h}}}\right)^{t} \varepsilon^{K}\right)$ Eyh $<0$, o que $\operatorname{Eyh}^{2}-\sum_{t=0}^{T}\left(\frac{1}{1+\delta_{\mathrm{h}}}\right)^{t} \varepsilon_{\mathrm{h}}$ Eyh $+K_{b 0} \Pi_{b} \varepsilon_{\mathrm{h}}<0$ para que $\frac{\partial C_{h} t h}{\partial \beta}>0$. Como la expresión anterior representa una parábola que se abre hacia arriba, basta hallar sus raíces:

$\frac{\left.\sum_{t=0}^{T}\left(\frac{1}{1+\delta_{\mathrm{h}}}\right)^{t}\left|\varepsilon_{\mathrm{h}}\right|\right|^{2} \sqrt{\sum_{t=0}^{T}\left(\frac{1}{1+\delta_{\mathrm{h}}}\right)^{2 t} \varepsilon_{\mathrm{h}}^{2}-4\left(K_{b 0} \Pi_{h} \varepsilon_{\mathrm{h}}\right)}}{2}$ y $\frac{\sum_{t=0}^{T}\left(\frac{1}{1+\delta_{\mathrm{h}}}\right)^{t}\left|\varepsilon_{\mathrm{h}}\right|+\sqrt[2]{\sum_{t=0}^{T}\left(\frac{1}{1+\delta_{\mathrm{h}}}\right)^{2 t} \varepsilon_{\mathrm{h}}^{2}-4\left(K_{b 0} \Pi_{b} \varepsilon_{\mathrm{h}}\right)}}{2}$, donde $\left|\varepsilon_{\mathrm{h}}\right|$ es el valor absoluto de $\varepsilon_{\mathrm{h}}$. Es decir, Eyh debe estar entre esos límites para que la mayor valoración social del gasto entre los más impacientes lleve a un mayor recaudo mediante sus impuestos al consumo.

${ }_{12}$ Argentina, Bolivia, Brasil, Chile, Colombia, Costa Rica, Ecuador, E1 Salvador, Guatemala, Honduras, México, Nicaragua, Panamá, Paraguay, Perú, República Dominicana, Uruguay y Venezuela. 
Como se explica más adelante, como variables tributarias se usan las participaciones de ciertos impuestos en la PT. Si $\mathrm{I}_{\mathrm{k}}$ es el recaudo del impuesto $\mathrm{k}, \mathrm{k}=1, \ldots \mathrm{K}, \mathrm{y} \mathrm{PT}=\sum_{k=1}^{K} \mathrm{I}_{\mathrm{k}}$, la relación que se considera es $\% I_{k}=\frac{i_{k}}{P T}$. Por tanto, si el modelo fundamenta una relación entre $I_{k} y$ un parámetro $\theta$, tal que $\frac{\partial I_{k}}{\partial \theta}>(<) 0$ y $\frac{\partial I_{j k k}}{\partial \theta}=0$, como la asociación positiva entre impuestos al consumo y valoración social del gasto, es fácil establecer que $\frac{\partial \% I_{k}}{\partial \theta}(())$, hecho en que se basa la constatación que se propone.

Por supuesto, la segunda opción tiene riesgos que no se deben desconocer. Cuando se analizan datos agregados, su evolución no es necesariamente idéntica a la que predice el modelo microfundamentado, pues intervienen muchos factores que no se tienen en cuenta en el análisis teórico, como choques externos de flujos comerciales y de capitales ni la competencia tributaria en que participan los Estados para atraerlos, lo que exige incorporar algunas variables de control que se suelen emplear en la literatura empírica.

Además, es posible que el resultado para un grupo de contribuyentes no sea generalizable, al menos por la importancia del factor $\Pi_{b}$. Sin embargo, cuando se usan datos agregados, lo que resulta es el efecto total entre grupos, que no necesariamente coincide con lo que se predice para uno de ellos, aunque se puede establecer qué primará con base en características como la notable inequidad de la distribución del ingreso en la región. Por ello, se decidió considerar solo tres variables agregadas relacionadas con parámetros del modelo, que conforme a la proposición 3 permiten generalizar resultados sin recurrir a muchos supuestos sobre la ordenación social o las condiciones iniciales de la economía.

Tampoco se deben menospreciar los posibles errores del método empleado por Profeta, pues cuando se usa un estudio de caso (como las elecciones en Italia de 2004) para demostrar una tesis supuestamente general, la evidencia no es sólida; la prueba a favor del modelo teórico puede ser accidental o producto de circunstancias particulares. Es decir, no hay un método infalible, y hay que elegir considerando los pros y contras de cada opción y el alcance de la investigación, sobre todo cuando intervienen variables no observables.

Una vez mencionados los problemas del método empleado en este ejercicio empírico, cabe precisar los factores macroeconómicos que incluye el modelo. Para representar $\mathrm{Ey}_{\mathrm{h}}$, sin distinguir entre grupos, se toma el promedio móvil ponderado del PIB per cápita de tres años (PMPIB_per). Los pesos de ponderación son $1 / 6,2 / 6 \mathrm{y} 3 / 6$, que se asignan del dato más antiguo al más reciente; los tres años corresponden al entero del cociente entre el número de observaciones (558) y los años 
en que el PIB per cápita descendió en cualquier país de la muestra ${ }^{13}$. Es decir, se considera que las expectativas de los contribuyentes sobre la situación económica dependen en parte de su experiencia anterior, pese a la tendencia a olvidar, y que aunque en un periodo de recesión no todos los sectores de la sociedad son afectados por igual, hay un cambio general de sus expectativas de ingreso a la baja.

Asimismo, se considera que la dependencia de la PT de impuestos como el IVA tiene relación directa con las tasas al consumo de los votantes más impacientes, en general los grupos pobres y medios, pues gran parte del consumo de los ricos, concentrado en bienes durables, es excluida o recibe beneficios tributarios (devoluciones o deducciones de otros impuestos); a esto se suma el hecho de que los eventuales aumentos del impuesto a este tipo de consumo se dificultan por la preocupación acerca de sus costos en eficiencia. Con base en ello y en las proposiciones mencionadas -en especial la proposición 2- se espera que cuanto mayor sea el promedio móvil del PIB per cápita menor será la participación del IVA (\%IVA).

E1 gasto público social con respecto al PIB es una proxy del grado de valoración social del gasto ( $\beta$ ) porque representa en forma más visible la atención de necesidades primarias como la educación y la salud. Además, puede internalizar la evaluación de los contribuyentes sobre el desempeño del Estado si compensa en parte el pago de impuestos e indica que la relación entre Estado y ciudadanos no es solo coercitiva sino también de intercambio y reciprocidad. Una deficiencia de esta medida es que no capta la efectividad de la provisión pública de bienes y servicios, y por tanto no capta el hecho de que dos países no logran los mismos avances con el mismo gasto, y para ello habría que incluir otras variables de control. No obstante, conforme a la proposición 3 se espera una relación directa entre el gasto público y la participación del IVA y de los impuestos generales y específicos al consumo (ByS) en la PT.

La formación bruta de capital representa el proceso de acumulación; la proposición 2 sugiere que un mayor acervo de capital es una oportunidad potencial para que el gobierno aumente la carga tributaria mediante impuestos al consumo, de manera que se espera una asociación positiva entre esta variable y el recaudo de los ByS, aunque es difícil determinar si también se genera mayor dependencia de estos impuestos, pues el acervo de capital también puede aumentar la base gravable del ISR.

13 . PMPIB_per $=3 /{ }_{6}$ PIB $_{-}$per ${ }_{t}{ }^{2} /{ }_{6}$ PIB_per ${ }_{t-1}+{ }^{1} / 6$ PIB_per $r_{t-2}$. Los resultados no cambian significativamente si a cada valor se le da el mismo peso (1/3) o si se usa el PIB per cápita de cada año, sin calcular promedios móviles. 
E1 cuadro 1 presenta la lista de variables endógenas que se incluyen en el ejercicio econométrico y los "determinantes" que sugiere la literatura, con una breve descripción y la fuente de información. Pese a que el análisis de la PT no corresponde a la constatación del modelo teórico propuesto, se incluye para examinar si los resultados de otros trabajos son válidos en el contexto latinoamericano.

Cuadro 1

Variables utilizadas en el ejercicio econométrico

\begin{tabular}{|c|c|c|}
\hline Variable & $\begin{array}{l}\text { Definición } \\
\end{array}$ & Fuente \\
\hline $\begin{array}{l}\% B y S, \% I V A \\
\% I S R, I n d / D i r \\
\text { recaudo_ByS y PT }\end{array}$ & $\begin{array}{l}\text { Variables endógenas. \%ByS, \%IVA y \%ISR son las partici- } \\
\text { paciones de los ByS, el IVA y el ISR en la carga tributaria } \\
\text { (PT). Ind/Dir es la relación entre recaudo de impuestos } \\
\text { indirectos y directos, y recaudo_ByS es el recaudo por ByS } \\
\text { como proporción del PIB }\end{array}$ & $\begin{array}{l}\text { Cepal y mi- } \\
\text { nisterios de } \\
\text { economía }\end{array}$ \\
\hline $\begin{array}{l}\text { Caribe y Centroa- } \\
\text { mérica }\end{array}$ & $\begin{array}{l}\text { Variable dummy igual a } 1 \text { si un país pertenece al Caribe } \\
\text { o a Centroamérica. México no se incluyó en este, pues } \\
\text { su estructura económica se parece más a la de los países } \\
\text { suramericanos }\end{array}$ & $\begin{array}{l}\text { Elaboración } \\
\text { propia }\end{array}$ \\
\hline PMPIB_per & $\begin{array}{l}\text { Promedio móvil ponderado del PIB per cápita de tres } \\
\text { años (dólares constantes de 2000) }\end{array}$ & $\begin{array}{l}\text { Banco Mun- } \\
\text { dial (WDI) }\end{array}$ \\
\hline Tasacol & Tasa de interés de colocación & \\
\hline Desempleo(-1) & Rezago de la tasa de desempleo & \\
\hline Inf & Inflación, variación del IPC (\%) & \\
\hline Ayud_int & $\begin{array}{l}\text { Asistencia para el desarrollo y auxilios oficiales (dólares } \\
\text { de 2010) }\end{array}$ & \\
\hline Apertura & $\begin{array}{l}\text { Suma de las exportaciones y las importaciones (\% del } \\
\text { PIB) }\end{array}$ & \\
\hline Pib_crec & Crecimiento del PIB per cápita (\%) & \\
\hline Agric & Tamaño del sector agrícola (\% del PIB) & \\
\hline$\triangle$ Capital & Formación bruta de capital (\% del PIB) & \\
\hline Recursos_nat & Rentas por exportación de recursos naturales (\% del PIB) & \\
\hline Gasto_soc & $\begin{array}{l}\text { Gasto social (\% del PIB) } \\
\text { Población rural (\% de la población total) }\end{array}$ & $\begin{array}{l}\text { Cepal } \\
\text { Banco Mun- }\end{array}$ \\
\hline Pob_mayor & Población mayor de 65 años (\% de la población total) & dial (WDI) \\
\hline Dens_pob & Densidad poblacional (miles de personas por $\mathrm{km}^{2}$ ) & \\
\hline Choque & $\begin{array}{l}\text { Valor acumulado del producto en una crisis económi- } \\
\text { ca -valor absoluto- y poder del gobierno en el congreso } \\
\text { (número de escaños) }\end{array}$ & $\begin{array}{l}\text { Elaboración } \\
\text { propia }\end{array}$ \\
\hline Leg & $\begin{array}{l}\text { Variable dummy igual a } 1 \text { si hay elecciones legislativas en } \\
\text { el año respectivo }\end{array}$ & $\begin{array}{l}\text { Banco Mun- } \\
\text { dial (DPI) }\end{array}$ \\
\hline Ejec & $\begin{array}{l}\text { Variable dummy igual a } 1 \text { si hay elecciones presidenciales en el año } \\
\text { respectivo }\end{array}$ & \\
\hline Derecha, Izquierda & $\begin{array}{l}\text { Variables dummies iguales a } 1 \text { si el partido de gobierno es de } \\
\text { derecha o izquierda }\end{array}$ & \\
\hline Unicameral & Variable dummy igual a 1 si el congreso tiene una sola cámara & \\
\hline Democracia & $\begin{array}{l}\text { Índice de democracia igual al promedio de los índices de liberta- } \\
\text { des políticas y civiles. de } 1 \text { (máxima libertad) a } 7 \text { (mínima libertad) }\end{array}$ & $\begin{array}{l}\text { Freedom } \\
\text { House }\end{array}$ \\
\hline
\end{tabular}

Fuente: Castañeda (2012) y Dioda (2012), elaboración propia.

Aunque algunos trabajos recientes introducen algunas variables -en
especial de tipo político- que no se incluyen en el cuadro, como los
índices de corrupción o el grado de corresponsabilidad entre gober- 
nantes y ciudadanos, se excluyeron porque reducen notablemente el tamaño de la muestra (a unas 100 observaciones), pues su recopilación y sistematización es reciente (desde la segunda mitad de los noventa). Se corrieron algunas regresiones intermedias -que aquí no se presentan- que usaban este tipo de variables, pero sus coeficientes no resultaron estadísticamente significativos.

Las tres primeras variables endógenas del cuadro 1 (\%ByS, \%IVA y $\% I S R)$, como proporciones de los ingresos tributarios por impuestos generales y específicos al consumo, IVA e ISR, constituyen una innovación en relación con la literatura empírica; el uso del recaudo de cada fuente, por otro lado, puede sobrestimar la importancia de factores como el desarrollo económico (PIB per cápita), debido a su relación con la PT.

En este ejercicio se intenta evitar problemas de subespecificación o variable omitida y se incluye un conjunto de factores económicos, demográficos, sociales y políticos. Junto a PMPIB_per (en logaritmos para resolver problemas de escala y de posible heteroscedasticidad), Gasto_soc y $\Delta$ Capital, que relacionan esta constatación con el modelo teórico, se incluyen otras, como la tasa de interés de colocación en algunas especificaciones, que controla la restricción del crédito, de gran influencia en el consumo.

Las estimaciones primero se hicieron suponiendo efectos aleatorios; después se ajustaron por heteroscedasticidad ${ }^{14}$. Los modelos 1 y 3 del cuadro $2^{15}$ incorporan todas las variables presuntamente exógenas, sin importar su significancia; con un proceso iterativo de eliminación de las no relevantes se llegó a los modelos 2 y 4, y se encontró que el logaritmo natural de PMPIB_per se relaciona positivamente (negativamente) con la participación del ISR (ByS) en el recaudo, relación opuesta a la del gasto social, que en su conjunto coinciden con el pronóstico.

No obstante, el uso de errores estándar corregidos para panel (PCSE, por sus siglas en inglés) con estructuras autorregresivas y dummies específicas de cada país, considerando los problemas de autocorrelación serial y efectos fijos, arrojó un cambio de signo del coeficiente de Gasto_soc en el modelo 9, a diferencia de los modelos 3 y 4; aparte de ello se verifica que un mayor gasto social corresponde a una mayor participación de los ByS y del IVA en la PT, como indica el análisis teórico.

${ }^{14}$ Después se detectaron problemas de autocorrelación serial y de efectos fijos, usando las pruebas de Wooldridge y Hausman, respectivamente.

${ }^{15}$ Los modelos que se mencionan en adelante son los del cuadro 2 si no se indica lo contrario. 


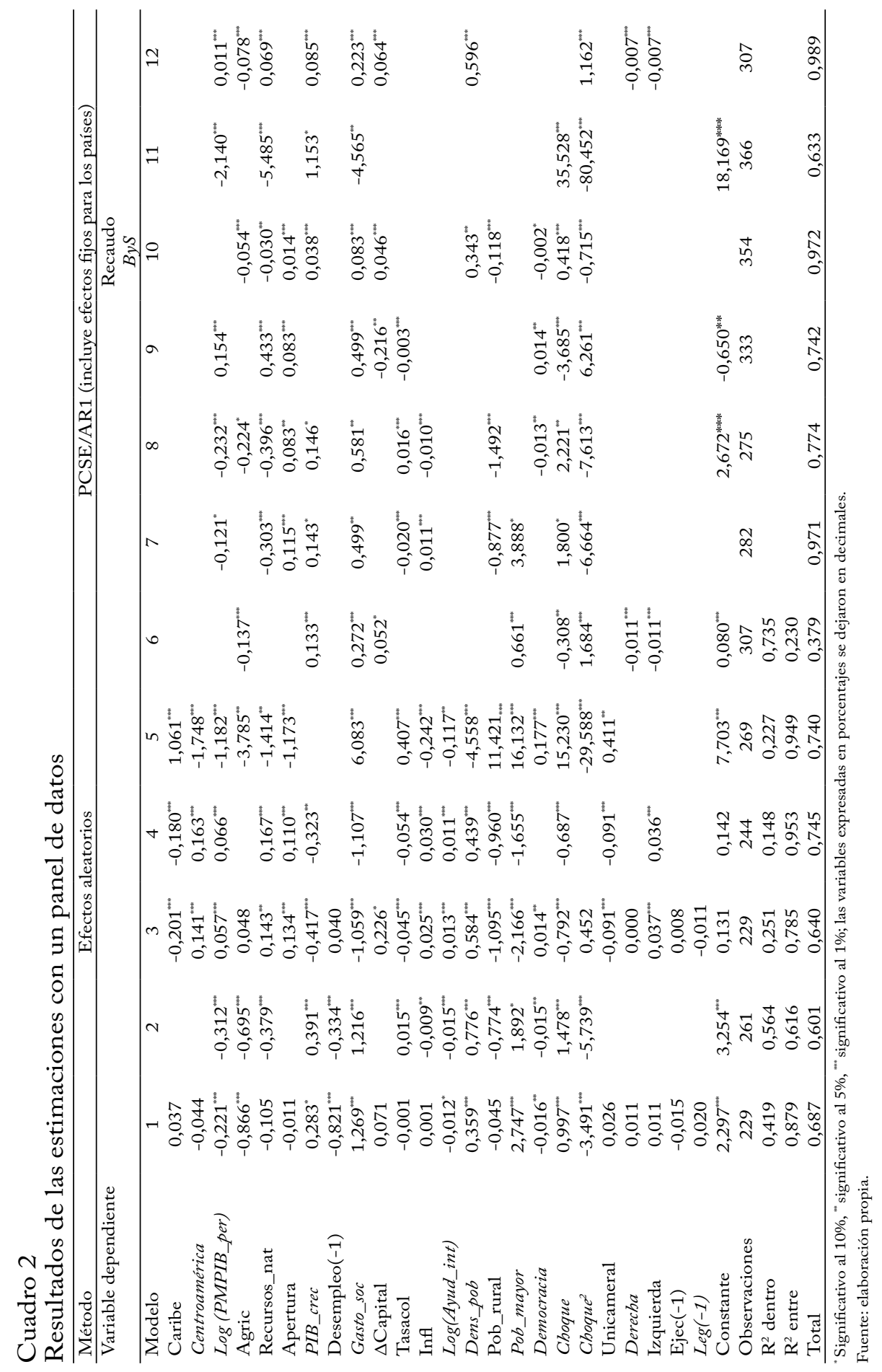

Revista de Economía Institucional, vol. i7, n.o 32 , Primer semestre/20i5, pp. I57-i 8 i 
En los modelos de efectos aleatorios la formación bruta de capital arroja una relación positiva con $\% I S R$, lo que no sucede con $\% B y S$, al menos de manera significativa; cabe recordar, sin embargo, que el modelo teórico permite asociar $\Delta$ Capital con el recaudo de impuestos al consumo y no con su participación en la carga tributaria. Esto llevó al modelo 10, donde se toma como variable endógena el recaudo por ByS, y se verifica su relación positiva con $\triangle$ Capital.

Tasacol, por su parte, se relaciona negativamente con $\% I S R$, pues una tasa de interés alta restringe el crédito para proyectos productivos y reduce los beneficios brutos de explotación para la economía en su conjunto. Los resultados de la asociación con ByS son ambiguos, pues el signo del coeficiente depende de las variables incluidas en cada modelo y del método de estimación. Sin embargo, la tasa de interés de colocación afecta en forma significativa a \%IVA en el modelo 7 , quizá debido a que este impuesto es general e influye particularmente en el consumo de las clases pobre y media, que son especialmente susceptibles a las tasas de crédito.

En cuanto al logaritmo de la ayuda internacional a proyectos de desarrollo social y económico (Ayuda_int), que se suelen tomar como sustitutos de los ingresos tributarios, se esperaría una relación negativa con la PT según buena parte de la literatura empírica (p. ej., Mahdavi, 2008), pero el cuadro 2 no lo constata. Esta variable sí es relevante para la estructura tributaria en los modelos de efectos aleatorios; estos recursos parecen desincentivar el uso de los ByS, y concentrar el recaudo en el ISR.

En lo que respecta a las variables demográficas, la densidad de población contribuye a que la administración tributaria reduzca los costos de recaudo y control de la evasión (Dioda, 2012), pero también parece contribuir a la concentración de la PT en los ByS y el ISR, a juzgar por los resultados de los modelos 1 a 4 . Una mayor proporción de adultos mayores se relaciona negativamente con $\% I S R$ (modelos 3 y 4 ), quizá por la exención del impuesto de renta a las pensiones en varios países de la región o a la reducción de ingresos que suele implicar la vejez, mientras que con \%Bys la relación es directa, un posible efecto indirecto de lo primero. El porcentaje de población rural, proxy de la informalidad y de las dificultades para gravar al sector agrario, tiene una relación con \%ByS, \%IVA y \%ISR, aun en las estimaciones PCSE/AR1.

En el campo político se usó el índice de democracia transformando la variable original (cuadro 1) para que un mayor valor correspondiera a una mejor evaluación ${ }^{16}$; Choque, que integra la ocurrencia de una crisis

${ }^{16}$ Los coeficientes respectivos del cuadro 2 están asociados a la variable Democracia. 
económica y el poder político del presidente ${ }^{17}$; una dummy igual a 1 para los congresos unicamerales; dos variables dicotómicas que captan la tendencia ideológica de cada gobierno; y dos dummies que indican si en el año respectivo hay elecciones ejecutivas o legislativas, para determinar la posible dependencia de la tributación de las promesas de campaña.

Tomando en conjunto las estimaciones con efectos aleatorios y PCSE/AR1, el grado de democracia se relaciona inversamente con la dependencia del recaudo de ByS e IVA, porque un entorno democrático da mayores oportunidades para que la sociedad esté debidamente representada en los órganos de decisión colectiva (el congreso). Pero este es uno de los pocos casos en que los coeficientes estimados en los modelos que explican \%IVA, \%ByS y \%ISR no son consistentes con los de Ind/Dir.

Choque no afecta en forma lineal a la dinámica tributaria, pero en ciertos niveles induce una mayor dependencia de los impuestos al consumo ${ }^{18}$, y se relaciona marginalmente con un aumento de la carga tributaria. Es decir, las crisis económicas son una oportunidad para introducir reformas, pero el grado en que se puede defender la meta de recaudo de un impuesto regresivo como el IVA (o los ByS) depende del poder de su promotor (el gobierno) en el legislativo, que aquí se mide por el número de escaños de su partido en el congreso, porque le permite presionar para que se aprueben sus proyectos y blindarlos ante objeciones o modificaciones que surjan en el tránsito legislativo. Este factor tiene en cuenta la dificultad del ejecutivo para introducir proyectos acordes al modelo teórico, cuando no comparte sus beneficios con los congresistas. El coeficiente negativo de Choque ${ }^{2}$ en los modelos que incluyen como variable dependiente a $\% B y S$ e $\% I V A$ se explica por la posibilidad de que una recesión económica sea tan profunda que el aumento de los impuestos al consumo no compense la reducción de las bases gravables. Pero una mayor participación del IVA o los ByS en la PT, después de aprobar una reforma durante una recesión, no necesariamente se relaciona con un aumento del recaudo total, de modo que solo hay una recomposición de la estructura tributaria, como parece haber sucedido en México, por ejemplo.

Unicameral solo es significativa en los modelos de efectos aleatorios, y sugiere que los países con congresos unicamerales tienden a

${ }_{17}$ E1 valor acumulado del producto entre profundidad de una crisis económica -en valor absoluto- y poder político del gobierno en la cámara de diputados, según el número de escaños de su partido. Se construyó desde 1980.

${ }_{18}$ Por ejemplo, en el modelo 1 ese nivel debe ser menor o igual a $0,136 \approx 1,056 /$ $(2 \times 3,88)$.

Revista de Economía Institucional, vol. i7, n.o 32 , Primer semestre/2oi5, pp. I57-i 8 i 
una estructura tributaria en la que el ISR es menos importante, lo que quizá obedezca a la menor dispersión y el menor número de actores con poder de veto, y a la consiguiente reducción de los costos de transacción asumidos por los grupos de interés que buscan beneficios tributarios en el componente directo.

Los resultados obtenidos para la tendencia ideológica y los años de elecciones no son concluyentes, al menos en lo que se refiere a la estructura tributaria. Los modelos de efectos aleatorios sugieren que con partidos de gobierno de izquierda hay mayor participación de los ISR en el recaudo, frente a los países donde gobiernan partidos de centro, pero esto no sucede con las estimaciones PCSE/AR1. Sin embargo, la carga tributaria tiene una relación negativa e inequívoca con la variable Izquierda, un resultado contraintuitivo, pues se esperaría que un gobierno de izquierda auspiciara políticas redistributivas y obtuviera un mayor nivel de recaudo. No obstante, esto encuentra fundamento teórico en el teorema del votante mediano, en los modelos de votación probabilística y en la aplicación del juego del coronel Blotto a problemas de tributación (Castanheira et al., 2012).

En cuanto a la clasificación regional que solo se incluye en los primeros 6 modelos para reducir la incidencia de las variables no observadas específicas de cada país, se encuentra que Centroamérica depende menos del IVA que Suramérica y República Dominicana (el único país del Caribe de la muestra). Además Centroamérica tiene un sistema menos regresivo, a juzgar por los resultados del modelo 5 .

Por último pero no menos importante, las rentas por explotación de recursos naturales guardan una relación positiva y significativa con \%ISR, aun en la estimación PCSE/AR1, lo que es consistente con el aumento de la renta gravable de las empresas públicas y privadas que se dedican a actividades primarias. La evolución de estas rentas en los últimos años es uno de los principales factores que inciden en el notable aumento del recaudo de impuestos directos en países como Ecuador, dados los altos precios de sus exportaciones en los mercados internacionales (Jiménez et al., 2010). El modelo 12 sugiere una relación positiva entre $P T$ y Recursos_nat, un resultado sobre el que no hay unanimidad; algunos autores concuerdan (Ghura, 1998) y otros encuentran una relación negativa o condicionan el efecto final a la calidad de las instituciones (Bornhorst et al., 2009; Botlhole, 2011).

Después de comentar los principales resultados, es necesario destacar que en este tipo de ejercicios puede haber problemas de endogeneidad, debidos, por ejemplo, a la incorporación de Gasto_soc en el modelo 6, pues una baja PT podría reducir el gasto a niveles modestos; 
y en los modelos 1 y 2, una baja dependencia de los ByS quizá refleje debilidades administrativas que perjudican la provisión pública de bienes y servicios. Por ello se consideró un conjunto de variables que sirvieran como instrumentos del gasto social. Para determinar si había algún problema de endogeneidad en el modelo 2 se tomó el índice de Gini como proxy de la demanda social de redistribución del ingreso, y la tasa bruta de matrícula en educación secundaria como factor que motiva la participación política y facilita la creación de programas sociales (Galston, 2001). En el modelo 6 se remplazó el índice de Gini por Pob_rural, pues la prueba de significancia de la regresión de la primera etapa mostró que el primer índice no era relevante.

Una vez definidos los instrumentos válidos para cada modelo se hizo la prueba de Hausman para verificar si se cumplían las hipótesis nulas de exogeneidad, pero ambas fueron rechazadas (con valores $\mathrm{p}$ de 0,003 y 0), lo que corroboró la existencia de problemas de endogeneidad. La prueba de restricciones sobreidentificadas de Sargan verificó la validez de los instrumentos (con valores p de 0,460 y 0,941). E1 cuadro 3 presenta las estimaciones de los modelos 2 y 6 .

Cuadro 3

Estimaciones de los modelos 2 y 6, MCO2E

\begin{tabular}{|c|c|c|}
\hline Variable dependiente & \%ByS & PT \\
\hline Gasto_soc & $1,294^{\text {***** }}$ & $0,599^{* * * *}$ \\
\hline Log PMPIB_per & $-0,211^{* * *}$ & \\
\hline Agric & $-0,124$ & $-0,079^{*}$ \\
\hline Recursos_nat & $-0,136$ & \\
\hline PIB_crec & 0,269 & $0,164^{* * * *}$ \\
\hline $\operatorname{Desempleo}(-1)$ & $-0,524^{* * * *}$ & \\
\hline Tasacol & $-0,113^{* *}$ & \\
\hline Infl & 0,021 & \\
\hline Pob_mayor & $3,064^{* * * *}$ & $-0,156$ \\
\hline $\log$ (Ayud_int) & $-0,008$ & \\
\hline Pob_rural & $-0,391^{* * * *}$ & \\
\hline Choque & $1,943^{* * * *}$ & $-0,337^{* *}$ \\
\hline Choque $^{2}$ & $-7,884^{* * * *}$ & $1,753^{\text {***** }}$ \\
\hline Democracia & $-0,027^{* * *}$ & \\
\hline Dens_pob & $0,850^{* * * *}$ & \\
\hline$\Delta$ Capital & & $0,051^{*}$ \\
\hline Derecha & & $-0,008^{* * * *}$ \\
\hline Izquierda & & $-0,010^{* * * *}$ \\
\hline Constante & $2,186^{* * * *}$ & $0,082^{* * * *}$ \\
\hline Observaciones & 146 & 244 \\
\hline $\mathrm{R}^{2}$ dentro & 0,484 & 0,673 \\
\hline $\mathrm{R}^{2}$ - entre & 0,834 & 0,483 \\
\hline Total & 0,720 & 0,540 \\
\hline
\end{tabular}

Si se relacionan las implicaciones del modelo teórico con los resultados del ejercicio empírico se pueden explicar algunos cambios estilizados 
de las estructuras tributarias latinoamericanas en los últimos años, y las diferencias entre países. A continuación se comentan dos de interés.

La tendencia regresiva de las reformas tributarias adoptadas en periodos de crisis, en las que ha primado el objetivo recaudatorio y el IVA ha sido una fuente importante para lograrlo (Castañeda, 2012), se puede entender como una decisión racional de los gobiernos que aumentan los impuestos al consumo, aunque las condiciones económicas de las clases pobre y media no sean las mejores o muestren un retroceso. Por tanto, los países de mayor grado de desarrollo, medido por el PIB per cápita -lo que supone que crecieron durante largos periodos o tuvieron épocas de bonanza-, tienden a presentar una estructura tributaria menos dependiente de los impuestos indirectos (cuadro 2).

Gráfica 1

Gasto público social en América Latina, 1990-2010

(Porcentaje del PIB)

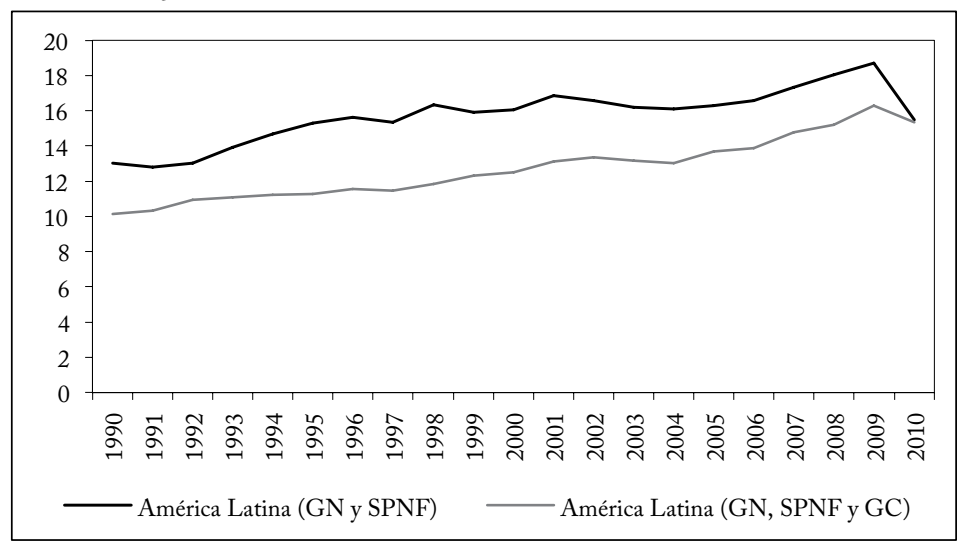

GN, SPNF y GC: gobierno nacional, sistema público no financiero y gobierno central, respectivamente. La primera serie incluye Argentina, Bolivia, Brasil, Colombia, Costa Rica, Panamá y Perú, por disponibilidad de datos; la segunda, todos los países incluidos en el ejercicio econométrico, además de Cuba, sin considerar el nivel de agregación.

Fuente: Cepal, elaboración propia.

Por otra parte, el análisis teórico pronostica que un gobierno aumentará los impuestos al consumo cuando mejora la valoración social del gasto; por ello, en sociedades excluyentes, como suelen serlo las latinoamericanas, si el gasto social aumenta y el Estado se hace razonablemente más visible, en la medida en que este factor tiene gran incidencia en las condiciones de vida de los ciudadanos, es razonable que se financie principalmente con impuestos indirectos (ver Castañeda, 2014, para el caso colombiano). La gráfica 1, que muestra el 
gasto social promedio sin ponderar de América Latina entre 1990 y 2010, indica que este ha crecido en general, aunque en algunos periodos es más evidente, como en la primera mitad de los noventa, y no es extraño que precisamente en ese lustro la PT haya pasado a depender más del IVA en buena parte de América Latina.

\section{CONCLUSIONES}

Las diferencias que suelen encontrar los estudios que comparan la estructura y el recaudo tributario entre países se pueden explicar, al menos en parte, por el uso que los gobiernos dan al ingreso fiscal, las condiciones materiales de los ciudadanos y sus expectativas sobre el desempeño económico. Existe un vínculo estrecho entre fuentes y usos; y el aumento de la presión tributaria, una tarea pendiente en la región, está limitado por la disposición de los contribuyentes a aceptar una mayor carga tributaria.

El modelo teórico que aquí se expuso sugiere que el aumento del gasto público social, como proxy del peso que los ciudadanos dan a la acción estatal en su bienestar, justifica el aumento de los impuestos al consumo, lo que lleva a una mayor dependencia de fuentes como el IVA. Por otra parte, y aunque parezca contraintuitivo, durante una crisis económica se pueden anticipar aumentos de esos impuestos, que por su naturaleza son regresivos y afectan especialmente a los contribuyentes con menor preferencia por el futuro, que suelen pertenecer a los deciles medios e inferiores de la distribución del ingreso.

La ideología política no muestra una relación significativa con la estructura tributaria e independiente de la técnica de estimación; las variables Izquierda y Derecha no son relevantes cuando se corrigen algunos problemas de correlación serial y heteroscedasticidad mediante PCSE/AR1. La acción de los gobiernos parece ser más sensible a factores económicos y demográficos, que delimitan las bases gravables. Esto no significa que la ideología no influya en la formulación de la política económica, sino que en el caso de la tributación en América Latina las condiciones externas que han enfrentado los gobiernos han limitado sus opciones a unas pocas, no siempre concordantes con promesas de campaña o principios de partido.

Es notable que el modelo que se desarrolla en este artículo -pese a estar anclado en la hipótesis del ingreso permanente, que suaviza el consumo en tiempos de calma relativa, certidumbre y pleno empleo- dé cuenta de algunas reformas adoptadas en América Latina en tiempos de crisis, en los que se restringe el crédito y la información es precaria. La decisión racional de un político, según el análisis teórico, 
es aumentar los impuestos al consumo de los grupos que ajustan a la baja el valor presente de sus ingresos y tienen baja disposición a esperar, lo que induce a recuperar o aumentar la PT durante una recesión concentrando los costos en los grupos de menor ingreso. Así, aunque en estas circunstancias las políticas públicas busquen recuperar el empleo o reactivar la producción, lo que suele justificar beneficios tributarios a la inversión, el aumento de los impuestos indirectos no solo es una decisión coyuntural y complementaria, sino que además puede ser útil electoralmente, y más si financia gasto social.

Sin embargo, este artículo, más que ser un trabajo terminado, busca motivar investigaciones con mayor grado de precisión que examinen, por ejemplo, estudios de caso que permitan incorporar especificidades de cada país, que, así compartan algunas características -como la desigualdad socioeconómica-, son muy heterogéneos, como lo indica la significancia estadística de los coeficientes de las variables de agrupación regional y la insuficiencia de los modelos de efectos aleatorios.

Asimismo, se debe señalar que el modelo teórico que aquí se desarrolla es incompleto, pues no considera el proceso de aprobación en el congreso, en el que se pueden hundir muchas iniciativas del gobierno, ni la sincronía entre el cambio de un parámetro y la decisión de política tributaria; por ejemplo, una reducción general de la valoración social del gasto llevaría a rebajar los impuestos al consumo, pero esto se demoraría en el mejor de los casos, pues hay compromisos presupuestales que no se pueden desatender (como la nómina del sector público), lo que además explica porqué son más frecuentes las alzas de impuestos que los recortes. En suma, se necesitan estudios de caso y de economía política que examinen a profundidad las condiciones que dan fundamento a los sistemas tributarios latinoamericanos y a sus continuas reformas.

\section{REFERENCIAS}

1. Besley, T. y T. Persson. "The origins of State capacity: Property rights, taxation, and politics", American Economic Review 99, 4, 2009, pp. 1218-1244.

2. Besley, T. y T. Persson. "State capacity, conflict, and development", Econometrica 78, 1, 2010, pp. 1-34.

3. Bornhorst, F.; S. Gupta y J. Thornton. "Natural resource endowments and the domestic revenue effort", European Journal of Political Economy 25, 4, 2009, pp. 439-446.

4. Botlhole, T. "Natural resources, institutions and tax revenue mobilization in sub-Sahara Africa", comunicación presentada en la Conferencia sobre Economía Aplicada, Kastoria, Instituto Tecnológico de Macedonia Occidental, 2011. 
5. Castanheira, M.; G. Nicodeme y P. Profeta. "On the political economics of tax reforms: Survey and empirical assessment", International Tax and Public Finance 19, 4, 2012, pp. 598-624.

6. Castañeda, V. "Una revisión de los determinantes de la estructura y el recaudo tributario: el caso latinoamericano tras la crisis de la deuda externa”, Cuadernos de Economía 57, 2012, pp. 77-112.

7. Castañeda, V. "El gasto social como factor que favorece una mayor dependencia del IVA. Un análisis para el caso colombiano", Ecos de Economia 38, 2014, pp. 185-215.

8. Coughlin, P. Probabilistic voting theory, Cambridge, Mass., Cambridge University Press, 1992.

9. Dioda, L. Structural determinants of tax revenue in Latin America and the Caribbean: 1990-2009, México D. F., Cepal, 2012.

10. Friedman, M. A theory of consumption function, Princeton, Princeton University Press, 1957.

11. Galli, E. y P. Profeta. "The political economy of tax complexity", Pavia, Società Italiana di Economia Pubblica, 2007.

12. Galston, W. "Political knowledge, political engagement, and civic education", Annual Review of Political Science 4, 2001, pp. 217-234.

13. Ghura, D. "Tax revenue in sub-Sahara Africa: Effects of economic policies and corruption", working paper, Washington, IMF, 1998.

14. González, J. y A. Pecha. “Tasa de preferencia intertemporal, equilibrio y estabilidad en los modelos de crecimiento neoclásico”, Cuadernos de Economía 32, 2000, pp. 61-76.

15. Hettich, W. y S. Winer. "Economic and political foundations of tax structure”, American Economic Review 78, 4, 1988, pp. 701-712.

16. Hettich, W. y S. Winer. "The political economy of taxation”, C. Muller, ed., Perspectives on Public Choice, Cambridge, Cambridge University Press, 1997, pp. 481-505.

17. Hettich, W. y S. Winer. Democratic choice and taxation, Cambridge, Cambridge University Press, 1999.

18. Jiménez, J.; J. Gómez y A. Podestá, comps., Evasión y equidad en América Latina, Santiago de Chile, Cepal, 2010.

19. Mahdavi, S. "The level and composition of tax revenue in developing countries: Evidence from unbalanced panel data", International Review of Economics and Finance 17, 4, 2008, pp. 607-617.

20. Persson, T. y G. Tabellini. Political economics. Explaining economic policy, Cambridge, MIT Press, 2000.

21. Persson, T. y G. Tabellini. "Political economics and public finance", A. Auerbach y M. Feldstein, eds., Handbook of Public Economics, vol. III, Amsterdam, Elsevier, 2002.

22. Profeta, P. "Political support and tax reforms with an application to Italy", Public Choice 131, 2007, pp. 141-155.

23. Tanzi, V. y H. Zee. "Tax policy for emerging markets: developing countries", National Tax Journal 53, 2000, pp. 299-322.

24. Torgler, B. "Tax morale in Latin America", Public Choice 122, 1-2, 2005, pp. 133-157. 
25. Winer, S. "Normative public finance for political economists", comunicación presentada en la reunión de la Sociedad Europea de Elección Pública, Belgirate, Italia, 2002.

26. Winer, S. y W. Hettich. "The political economy of taxation: Positive and normative analysis when collective choice matters", working paper, Ottawa, Carleton University, 2003. 